\title{
Nucleon Sigma Terms from Lattice QCD
}

\author{
Lukas Varnhorst* for the Budapest-Marseille-Wuppertal Collaboration \\ Bergische Universität Wuppertal \\ E-mail: l.varnhorstet-online.de
}

\begin{abstract}
The nucleon-sigma-terms are measures of the light-quark- and strange-quark-content of the nucleon. Especially the stangeness-content is of significant interest for dark-matter searches, as it determines the coupling of several dark matter candidates to hadronic matter. While the sigmaterms can not be measured directly they can be determined via lattice QCD from first principles. The sigma-terms are related to the light- and strange-quark mass dependence of the nucleon mass by the Feynman-Hellmann-theorem. To measure this dependence we used $N_{f}=1+1+1+1$ ensembles generated with tree-level improved Symmanzik gauge action and tree-level improved clover Wilson fermions with three levels of HEX smearing at four values of the lattice spacing.
\end{abstract}

The 33rd International Symposium on Lattice Field Theory

14 -18 July 2015

Kobe International Conference Center, Kobe, Japan*

${ }^{*}$ Speaker. 


\section{Introduction}

The nucleon sigma terms are defined by the relations

$$
\sigma_{\pi N}=m_{u d}\langle N|\bar{u} u+\bar{d} d| N\rangle \quad \text { and } \quad \sigma_{\bar{s} s N}=2 m_{s}\langle N|\bar{s} s| N\rangle .
$$

Here $|N\rangle$ is a nucleon state and $u, d$ and $s$ are the respective quark fields. Despite the appearance of the quark masses $m_{q}$ in their definitions they are renormalization scheme independend quantities. They are a convenient way to parametrize the scalar quark content of the nucleon. The sigma terms cannot be measured directly in experiments but are of significant interest. One prominent example is the search for dark matter. Here the sigma terms enter as they allow to relate couplings at the quark level to couplings to the nucleon.

There have been several previous computations of the nucleon sigma terms [1-17]. Some of them are based on phenomenology, others use lattice QCD. An overview of these studies can be found in figure 1.
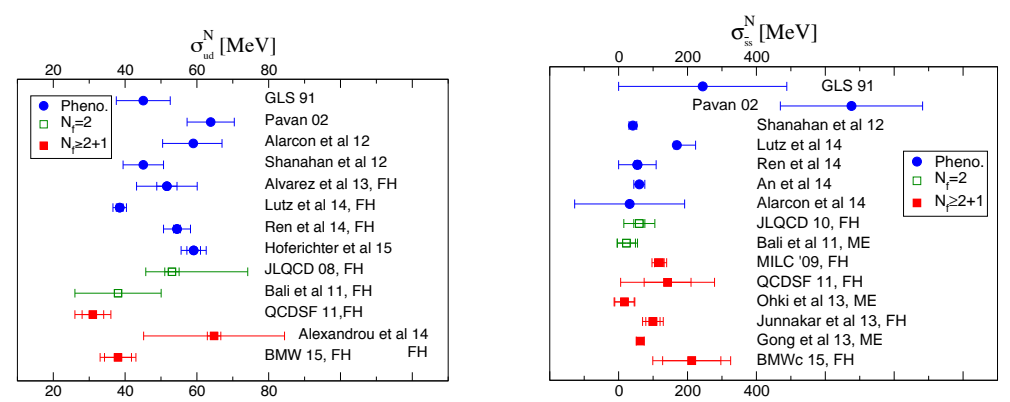

Figure 1: An overview of the estimates of $\sigma_{\pi N}$ and $\sigma_{\bar{s} s N}$ found in the literature.

This proceedings describes an ongoing study of the nucleon sigma terms on a set of $N_{f}=$ $1+1+1+1$ ensembles. These ensembles where generated with a tree-level improved Symmanzik gauge action and a tree-level improved Clover-Wilson fermion action with 3 iterations of HEX smearing. Details on these ensembles can be found in [19]. They feature 4 lattice spacings ranging from $0.102 \mathrm{fm}$ to $0.064 \mathrm{fm}$.

\section{Analysis}

To extract the sigma terms the Feynman-Hellman theorem is used. It relates the sigma terms to the derivative of the nucleon mass with respect to the quark masses via

$$
\sigma_{\pi N}=\left.m_{u d} \frac{\partial M_{N}}{\partial m_{u d}}\right|_{m_{s}} \text { and } \sigma_{\bar{s} s N}=\left.2 m_{s} \frac{\partial M_{N}}{\partial m_{s}}\right|_{m_{u d}} .
$$

It is interesting to note that, while in the above formula quark masses appears, which must be proper renormalized, the sigma-term itself requires no renormalization. One way to proceed is to replace the above derivatives with derivatives with respect to $M_{\pi}^{2}$ and $M_{\bar{s} s}^{2}:=\frac{1}{2}\left(M_{K^{0}}^{2}+M_{K^{+}}^{2}-M_{\pi}^{2}\right)$. In leading order chiral perturbation theory one finds $m_{u d} \propto M_{\pi}^{2}$ and $m_{s} \propto M_{\bar{s} s}^{2}$. Therefore one has the relations

$$
\sigma_{\pi N} \approx \Delta_{\pi}=\left.M_{\pi}^{2} \frac{\partial M_{P}}{\partial M_{\pi}^{2}}\right|_{M_{\bar{s}}^{2}} \text { and } \frac{\sigma_{\bar{s} s}}{2} \approx \Delta_{\bar{s} s}=\left.M_{\bar{s} s}^{2} \frac{\partial M_{P}}{\partial M_{\bar{s}}^{2}}\right|_{M_{\bar{\pi}}^{2}} .
$$


In this work the (tiny) error which is introduced by this approach is corrected. This is achieved by a non-perturbativley defined transformation matrix which relates the above derivatives to the derivatives of $M_{N}$ with respect to the quark masses. This matrix can be determined without the need for explicit renormalization factors.

The calculation proceeds in two steps. In the first step the dependence of nucleon mass on the meson masses is determined. In a second step the transformation matrix is determined which makes the analysis exact.

\subsection{The proton mass as a function of meson masses}

In order to fit any model to the lattice data baryon and meson masses must be extracted from the correlation functions measured on the lattice. This is done by fitting the correlation functions with the standard ansatzes

$$
C(t)= \begin{cases}A \cosh \left(-m\left(t-N_{t} / 2\right)\right) & \text { for mesons } \\ A \sinh \left(-m\left(t-N_{t} / 2\right)\right) & \text { for baryons. }\end{cases}
$$

starting at an appropriate timelag $t_{\min }$. The proper value of $t_{\min }$ is not straight forward to determine: If it is chosen to small then the extracted mass is affected by excited state contributions. If however $t_{\min }$ is chosen to large one has to face increasing statistical fluctuation which deteriorate the precision of the extracted masses. In this work the correlation function from all our ensembles are repeatedly fitted with different values of $t_{\min }$ in physical units. For each ensemble the quality of fit $Q$ was calculated. In the ideal case where the fitted model fully describes the observed data one would expect the $Q$-values to be uniformly distributed between 0 and 1 . This can be tested with a Kolmogorov-Smirnov test. From the $Q$-values of all ensembles the cumulative distribution function $F_{\text {obs }}$ of the underlying distribution can be estimated. It can be compared with the expected cumulative distribution function $F_{\exp }(x)=x$. This is done by calculating the maximal distance

$$
D=\sup _{x}\left|F_{\text {obs }}(x)-F_{\text {exp }}(x)\right| .
$$

The probability $P$ that $D$ is bigger then the observed $D$ is given by a well known function [18]. For each channel $t_{\min }$ was chosen so that $P>0.3$. For two channels the behavior of $F_{\mathrm{obs}}(x)$ as a function of $t_{\min }$ is illustrated in figure 2. A very similar approach was used in [19].
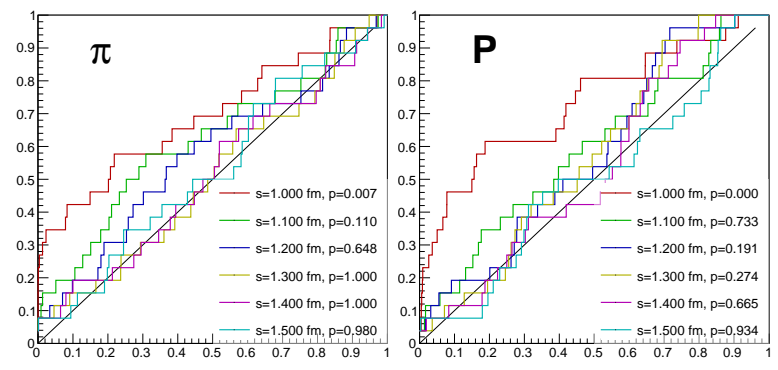

Figure 2: The behavior of the cumulative distribution function of the fit qualities as a function of $t_{\min }$ for the pion and proton channels.

For the scale setting $M_{\Omega}$ as a function of $M_{\pi}^{2}$ and $M_{\bar{s} s}^{2}$ was fitted to determine the lattice spacing at the physical point. To estimate the systematic error an additional scale setting with the nucleon 
mass instead of $M_{\Omega}$ was performed. Throughout the analysis a mass independent scale setting is adopted meaning that the lattice spacing depends solely on the gauge coupling $\beta$ and not on the quark mass parameters.

The nucleon can show a dependence on both the $M_{\pi}^{2}$ and $M_{\overline{S S}}^{2}$ mass, the lattice spacing $a$ and the finite volume. Also since in the ensembles used in this work $m_{u} \neq m_{d}$ a dependence on $M_{K^{+}}-M_{K^{0}}$ was allowed. Expanding the nucleon mass around the physical point on gets the leading terms

$$
\begin{array}{r}
M_{P}=\left(c_{0}+c_{1}\left(M_{\pi}^{2}-M_{\pi}^{(\phi) 2}\right)+c_{2}\left(M_{\overline{s s}}^{2}-M_{\bar{s} s}^{(\phi) 2}\right)+c_{3}\left(M_{K^{+}}-M_{K^{0}}\right)+c_{4} C(\beta)+\ldots\right) \\
\left(1+c_{5} F\left(M_{\pi}, L\right)\right) .
\end{array}
$$

Here ... is meant to indicate higher order terms that can possibly contribute to the nucleon mass away from the physical point. To estimate systematic errors introduced by such higher order terms the lattice data was fitted with several variants of this fit function. These variants where constructed in the following way:

- Continuum extrapolation is done with either $C(\beta)=a^{2}(\beta)$ or $C(\beta)=\alpha_{s}(\beta) a(\beta)$. Formally the action used has $\alpha_{s} a$ cutoff effect as it is a tree-level improved action, however often $a^{2}$ cutoff effects are numerical dominant.

- The next-to-leading term in $M_{\pi}^{2}$ is assumed to be either $M_{\pi}^{4}-M_{\pi}^{(\phi) 4}$ which is the next term in a taylor expansion or $M_{\pi}^{3}-M_{\pi}^{(\phi) 3}$ which is motivated by chiral perturbation theory.

- Finite volume effects are parametrized via $F(L)=\sqrt{\frac{M_{\pi}}{L^{3}}} e^{-M_{\pi} L}$ or via $F(L)=e^{-M_{\pi} L}$

Here and throughout the analysis whenever there was no obvious different choice for a term of the form $c_{i} X$ the contributions of higher order term where estimated by removing the term from the fit function and multiplying the whole function by either $1+c_{i} X$ or $1 /\left(1-c_{i} X\right)$. The fits where performed with three different pion mass cuts. All fits in this analysis are performed with a fully correlated $\chi^{2}$.

Once the nucleon has been fitted with a suitable fit function one can determine $\Delta_{\pi}$ and $\Delta_{\bar{s} s}$ and chiral perturbation theory could be used to estimate the sigma terms.

\subsection{The quark mass dependence}

Before the above derivatives can be transformed into quark mass derivatives the quark masses have to be defined in a proper way. For the method a definition of quark masses is needed which exhibits only multiplicative renormalization. Such a definition is given by the ratio-difference quark mass as described in [20]. This method combines the PCAC quark masses and the bare Wilson quark masses. For quarks of two flavors $i$ and $j$ the ratio of masses $r_{i j}$ and the difference of masses $d_{i j}$ are defined via

$$
r_{i j}=\frac{m_{i}^{P C A C}}{m_{j}^{P C A C}} \quad \text { and } \quad d_{i j}=a\left(m_{i}^{W}-m_{j}^{W}\right) .
$$

where $m_{i}^{W}$ is the bare Wilson quark mass and $m_{i}^{P C A C}$ is the PCAC quark mass. From these quantities one can define the ratio-difference quark mass as

$$
a \hat{m}_{i}^{r d}=\frac{1}{Z_{S}} a m_{i}^{r d}=\frac{1}{Z_{S}} \frac{r_{i j} d_{i j}}{r_{i j}-1} \text { and } a \hat{m}_{j}^{r d}=\frac{1}{Z_{S}} a m_{j}^{r d}=\frac{1}{Z_{S}} \frac{d_{i j}}{r_{i j}-1} .
$$


Here $m_{i}^{r d}$ corresponds to the unrenormalized quark mass while $\hat{m}_{i}^{r d}$ denotes the renormalized quark mass. These definition can be tree-level improved by a minor modification which is detailed in [20]. In the above formulae $Z_{S}$ is the scalar renormalization factor which has to be determined by another method. However in the case of the sigma terms the renormalization factor cancels out. To evaluate the necessary derivatives it suffices to define a ratio

$$
R_{i}=\frac{a m_{i}^{r d}}{a m_{i}^{r d,(\phi)}}=\frac{\hat{m}_{i}^{r d}}{\hat{m}_{i}^{r d,(\phi)}}
$$

Here $\hat{m}_{i}^{r d,(\phi)}$ denotes the renormalized quark mass at the physical point. The quantity $a m_{i}^{r d,(\phi)}$ is defined separately for each value of the lattice spacing and corresponds to the value of the unrenormalized ratio-difference quark mass at this lattice spacing at the physical point. Both definition of the ratios agree in a mass independent renormalization scheme. In practice $a m_{i}^{r d,(\phi)}$ is determined by a fit to the quark masses with an ansatz of the from

$$
\begin{aligned}
& a m_{i}^{r d}=c_{m}(a)\left(1+c_{0}\left(M_{\pi}^{2}-M_{\pi}^{(\phi) 2}\right)+c_{1}\left(M_{\bar{s} s}^{2}-M_{\bar{s} s}^{(\phi) 2}\right)+c_{2}\left(M_{K^{+}}-M_{K^{0}}\right)\right.+\ldots) \\
&\left(1+c_{3} F\left(M_{\pi}, L\right)\right)
\end{aligned}
$$

where $c_{m}$ is a separate fit parameter for each lattice spacing and ... is meant to correspond to higher order corrections along the lines of those described in the previous section. From this fit

$$
R_{i}=\frac{a m_{i}^{r d}}{c_{m}(a)}
$$

can be extracted. To use the information from this fit function to determine the sigma terms one has to define a matrix

$$
J=\left(\begin{array}{ll}
\left.\frac{m_{u d}}{M_{\pi}^{2}} \frac{\partial M_{\pi}^{2}}{\partial m_{u d}}\right|_{m_{s}} & \frac{m_{u d}}{M_{s s}^{2}} \frac{\partial M_{s s}^{2}}{\partial m_{u d}} \\
\left.\frac{m_{s}}{M_{\pi}^{2}} \frac{\partial M_{\pi}^{2}}{\partial m_{s}}\right|_{m_{u d}} & \left.\frac{m_{s}}{M_{s s}^{2}} \frac{\partial M_{s s}^{2}}{\partial m_{s}}\right|_{m_{u d}}
\end{array}\right)
$$

which fulfills

$$
\left(\begin{array}{ll}
\sigma_{\pi N} & \sigma_{\bar{s} s} / 2
\end{array}\right)^{T}=J\left(\begin{array}{ll}
\Delta_{\pi} & \Delta_{\overline{s s}}
\end{array}\right)^{T}
$$

The inverse of $J$ can be easily determined from eq. (2.9) using

$$
\left(J^{-1}\right)_{i j}=\left.M_{j} \frac{\partial R_{i}\left(M_{\pi}^{2}, M_{\bar{s} s}^{2}\right)}{\partial M_{j}^{2}}\right|_{M_{k}^{2}, k \neq j} .
$$

The elements of the matrix $J$ from one representative fit can be found in figure 3. The resulting matrix is dominated by the diagonal entries which are close to one. The off-diagonal elements which are much smaller then the diagonal components are describing the mixing between $\left(\Delta_{\pi}, \Delta_{\bar{s} s}\right)$ and $\left(\sigma_{\pi N}, \sigma_{\bar{s} s N}\right)$.

\section{Results}

To estimate the systematic error different versions of all fit functions where employed to estimate contributions of higher order terms. Also the entire analysis have performed with three different 


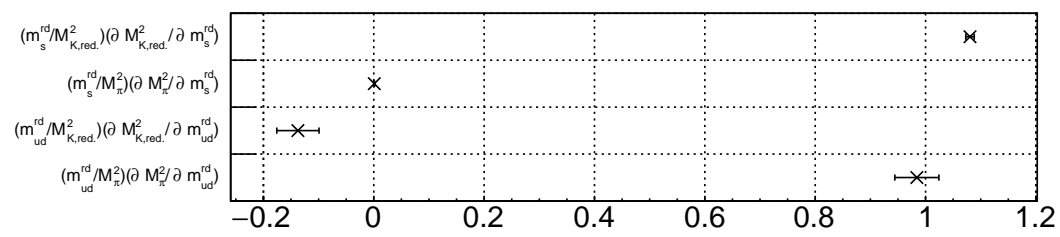

Figure 3: The elements of the matrix $J$ described in the main text from one representative fit. The errors are purely statistical.

pion cuts. The scale setting has been done both with the $\Omega$ mass and with the proton mass itself. All together this resulted in 864 different fit procedures which where combined into a histogram. The analyses where wighted once with the Akaike-weight as described in [19] and with a uniform weight. The spread was taken as the systematic error. The statistical error was estimated by repeating the entire analysis, including the 864 different modifications, on 1000 bootstrap samples. The combined error was estimated by quadratically adding the systematic and the statistic error.

Before the final numbers can be given the analysis still need to be finalized and a independent crosscheck by a different member of our collaboration has to be performed. Both $\sigma_{u d}$ and $\sigma_{\bar{s} s N}$ show total error of $\sim 15 \%$. Histograms of all the performed analysis can be found in figure 4 .

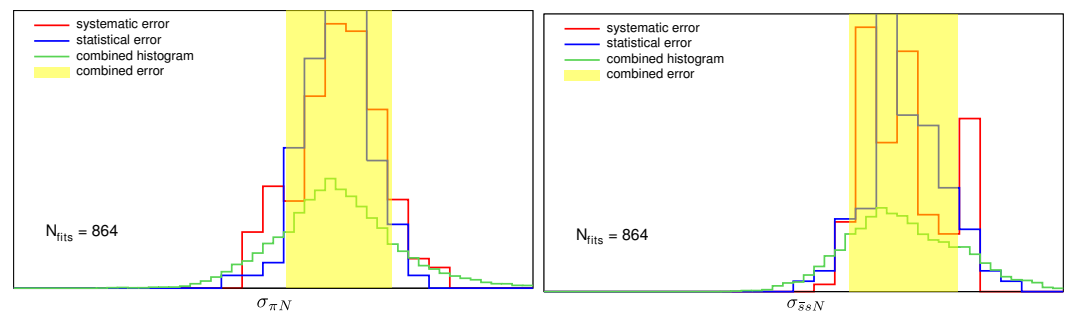

Figure 4: Preliminary histograms of the analysis. The left side corresponds to a value of $0 . x$-scales are different. There are three histograms: red: Histogram of the central value for each of the 864 analysis. blue: Histogram of the bootstrap samples of the mean of the 864 analysis. green: Combined histogram of all bootstrap samples from all analysis. The yellow bar indicates the total error.

\section{Acknowledgements}

Computations were performed using the JUGENE installation of FZ Jülich and HPC resources provided by GENCI-IDRIS (grant 52275), as well as further resources at FZ Jülich and clusters at Wuppertal and CPT. This work was supported in part by the OCEVU Labex (ANR-11-LABX0060), the A*MIDEX project (ANR-11-IDEX-0001-02) and by DFG grant SFB/TRR-55.

\section{References}

[1] J. Gasser, H. Leutwyler, and M. Sainio, Sigma-term update, Phys. Lett. B253 (1991) 252.

[2] M. Pavan, I. Strakovsky, R. Workman, and R. Arndt, The pion-nucleon Sigma term is definitely large: results from a G.W.U. analysis of pion nucleon scattering data, PiN Newslett. 16 (2002) 110, [hep-ph/0111066]. 
[3] J. M. Alarcon, J. Martin Camalich, and J. A. Oller, The chiral representation of the $\pi N$ scattering amplitude and the pion-nucleon sigma term, Phys.Rev. D85 (2012) 051503, [hep-ph/1110.3797].]

[4] L. Alvarez-Ruso, T. Ledwig, J. Martin Camalich, and M. J. Vicente-Vacas, The nucleon mass and pion-nucleon sigma term from a chiral analysis of lattice QCD world data, Phys. Rev. D88 (2013) 054507 [hep-ph/1304.0483].

[5] X.-L. Ren, L.-S. Geng, and J. Meng, Scalar strangeness content of the nucleon and baryon sigma terms, Phys. Rev. D91 (2014) 051502 [hep-ph/1404.4799].

[6] M. Lutz, R. Bavontaweepanya, C. Kobdaj, and K. Schwarz, On finite volume effects in the chiral extrapolation of baryon masses, Phys. Rev. D90 (2014) 054505 [hep-lat/1401 . 7805].

[7] C. Alexandrou, et al. (ETM Collaboration), The low-lying baryon spectrum with two dynamical twisted mass fermions, Phys. Rev. D80 (2009) 114503 [hep-lat/ 0910 . 2419].

[8] D. Toussaint and W. Freeman (MILC), The strange quark condensate in the nucleon in $2+1$ flavor QCD, Phys. Rev. Lett. 103 (2009) 122002 [hep-lat/ 0905.2432 ].

[9] R. Babich, R. C. Brower, M. A. Clark, et al., Exploring strange nucleon form factors on the lattice, Phys. Rev. D85 (2012) 054510 [hep-lat/1012. 0562].

[10] G. S. Bali, et al. (QCDSF Collaboration), The strange and light quark contributions to the nucleon mass from Lattice QCD, Phys. Rev. D85 (2012) 054502 [hep-lat/1111. 1600 ].

[11] R. Horsley, et al. (QCDSF-UKQCD Collaborations), Hyperon sigma terms for $2+1$ quark flavours, Phys. Rev. D85 (2012) 034506 [hep-lat/1110 . 4971].

[12] S. Durr, Z. Fodor, T. Hemmert, C. Hoelbling, J. Frison, et al. (BMW Collaboration), Sigma term and strangeness content of octet baryons, Phys. Rev. D85 (2012) 014509 [hep-lat/1109.4265].

[13] W. Freeman et al. (MILC Collaboration), The intrinsic strangeness and charm of the nucleon using improved staggered fermions, Phys. Rev. D88 (2013) 054503 [hep-lat/1204 . 3866].

[14] P. Junnarkar, and A. Walker-Loud, The Scalar Strange Content of the Nucleon from Lattice QCD, Phys. Rev. D87 (2013) 114510 [hep-lat/1301.1114].

[15] M. Gong, et al. (XQCD), Strangeness and charmness content of nucleon from overlap fermions on 2 + 1-flavor domain-wall fermion configurations, Phys. Rev. D88 (2013) 014503 [hep-ph/1304.1194].

[16] C. Alexandrou, V. Drach, K. Jansen, C. Kallidonis, and G. Koutsou, Baryon spectrum with $N_{f}=2+1+1$ twisted mass fermions, Phys. Rev. D90(7) (2014) 074501 [hep-lat/1406.4310].

[17] S. Durr et al., Lattice computation of the nucleon scalar quark contents at the physical point, arXiv:1510.08013 [hep-lat].

[18] W. H. Press, S. A. Teukolsky, W. T. Vetterling, B. P. Flannery, Numerical Recepies in C: The Art of Scientific Computing, Cambridge University Press, 1992

[19] S. Borsanyi et al., Ab initio calculation of the neutron-proton mass difference, Science 347 (2015) 1452 [arXiv:1406.4088 [hep-lat]].

[20] S. Durr et al., Lattice QCD at the physical point: Simulation and analysis details, JHEP 1108 (2011) 148 [arXiv:1011.2711 [hep-lat]]. 